Kerstin Dingfeld, Lara Fricke und Franz Vergöhl

\title{
Lehr- und Lernräume für Studierende gestalten
}

\section{Anforderungen und Perspektiven}

Die Förderung von studentischer Partizipation in der Hochschullehre ist eine Herausforderung für die Hochschulen. Aus einer studentischen Perspektive ergeben sich verschiedene Anforderungen, um durch Lehr- und Lernräume positiv auf studentische Partizipation einzuwirken. Im vorliegenden Beitrag sollen Vorstellungen $\mathrm{zu}$ zukünftiger Hochschullehre skizziert werden. Dabei richtet sich der Blick vor allem darauf, welche Lehr- und Lernräume sich Studierende wünschen.

\section{Kontexte}

Lernräume lassen sich als „Umgebungen, die Erwachsene zum Zweck des Lernens zeitlich begrenzt aufsuchen“, beschreiben (Siebert 2006, 21). Dies kann beispielsweise ein Seminarraum, eine Ausstellung oder eine Bibliothek sein (Siebert 2006, 21). Diese Perspektive umfasst sowohl Räume für Lehrveranstaltungen als auch Räume für das Selbststudium von Lernenden. In einer zukunftsfähigen Hochschulinfrastruktur ist eine Unterscheidung zwischen Lehrund Lernraum nicht notwendig, da Bildung - und das ist schließlich das, worum es am Ende geht - immer sowohl durch Lehre als auch Lernen entsteht. Für das studentische Lernen sind Lernumgebungen wichtig und effektiv, in denen die notwendigen Ressourcen zur Verfügung gestellt werden, die es ermöglichen, sich akademisch und sozial zu integrieren (Mayrberger 2017, 114).

Hochschulen präsentieren sich gerne als Orte mit einer besonders lernförderlichen Atmosphäre. Die Leuphana Universität verspricht zum Beispiel „eine Atmosphäre zu schaffen, die durch gelebte Diversität und gegenseitige Wertschätzung inspirierend wirkt“ (Leuphana 2019). Die Universität Hamburg sieht sich als Ort der Offenheit des Zugangs zu Bildung und Wissenschaft (Universität Hamburg 2018). Um solchen Ansprüchen gerecht zu werden, sollte die Hochschule ein Ort sein, an dem alle Studierenden, Lehrenden sowie Besucherinnen und Besucher teilhaben können. Innen- und Außenräume müssen unterschiedlichen Bedürfnissen und Anforderungen entsprechen (Günter/Rauber 2019, 4). 
Die Gebäude und Räume zum Lehren und Lernen sollten barrierefrei sein, damit sie auch von Menschen mit Behinderungen, mit jedweder Form von Beeinträchtigung, ,in der allgemein üblichen Weise, ohne besondere Erschwernis und grundsätzlich ohne fremde Hilfe auffindbar, zugänglich und nutzbar sind“ (§ 4 Gesetz zur Gleichstellung von Menschen mit Behinderungen).

In einer Veröffentlichung des Centrums für Hochschulentwicklung (CHE) wird ein Bild gezeichnet, in dem durch das ständige Anwachsen der Studierendenzahlen Hochschulen die Chance nutzen müssten, sich nach innen und nach außen zu profilieren (Ziegele et al. 2019). Dabei spielt die Entwicklung eines vielfältigen Systems eine zentrale Rolle, das als Ganzes der Vielfalt der Studierenden und der Vielfalt der Aufgaben der Hochschulen gerecht werden kann. Die Ausdifferenzierung der Hochschulen wird vermutlich auch räumliche Auswirkungen haben.

\section{Anpassung der Lehr- und Lernräume an Bedürfnisse}

Gleichzeitig sollen Hochschulen Lehrstrategien entwickeln, die neben klassischen Lehrformaten auch aktivierende Formen der Lehre enthalten. Hierzu zählen Ansätze des problem- und projektorientierten Lernens sowie die Vermittlung von innovativen Methodenkompetenzen wie Design Thinking (Stifterverband 2019, 26). Projektbasierte Lernmodule ermöglichen den Studierenden, kooperativ über die Grenzen der Hochschule hinaus zu denken und mit Partnerinnen und Partnern aus Wirtschaft und Zivilgesellschaft zusammenzuarbeiten (Stifterverband 2019, 27).

Darüber hinaus wird es in Zukunft vermutlich zu einer Digitalisierung vieler Lehr-Lernprozesse kommen. Da es trotzdem weiterhin physische, analoge Lehrund Lernräume an Hochschulen geben wird, werden mit diesen neuen Entwicklungen besondere Anforderungen an die Lehr- und Lernräume gestellt. Ein Beispiel für eine innovative Unterrichtsmethode ist der Flipped Classroom (,umgedrehter Unterricht“). Hierbei wird die Lehre umgedreht und die Lernenden bereiten das, was normalerweise im Unterricht gelehrt wird, bereits eigenständig vor. Dies passiert oftmals mithilfe von digitalen Tools. Der Flipped Classroom bietet den Vorteil, dass in der Präsenzlehre direkt tiefer in die Materie eingestiegen werden kann (Bergmann/Sams 2012, 13). Hierbei können die Grenzen zwischen Lehre und Lernen verschwimmen, wie oben bereits angedeutet. 
Um den Anforderungen dieser neuen Lehr-Lernformate gerecht zu werden, muss die Gestaltung von Lehr- und Lernräumen stets agil bleiben. Egal ob Räume nur für das Selbststudium oder für Lehrveranstaltungen genutzt werden, sollten sie so gestaltet sein, dass sie ein optimales Umfeld für studentische Partizipation schaffen. Die meisten Seminarräume sind beispielsweise so ausgerichtet, dass die Dozierenden vorne hinter einem separaten Tisch stehen beziehungsweise sitzen. Dies kann unter Umständen dazu führen, dass Hierarchien reproduziert werden, was nicht für jedes Lehr-Lern-Format von Vorteil ist. Noch ausgeprägter gestaltet sich dies in Vorlesungssälen. Ein weiterer wichtiger Aspekt ist die Größe des Raumes: Ist er zu groß, setzen sich Studierende häufig nach hinten und somit wird gute Kommunikation erschwert. Ist er zu klein, wirkt sich dies allerdings auch negativ auf die Lehr-Lern-Situation aus, indem die Teilnehmenden sich beispielsweise eingeengt fühlen oder die Sicht nach vorne behindert ist. Dies ist im folgenden Erfahrungsbericht weiterführend skizziert.

\section{Erfahrungsbericht}

Ich war auf dem Weg zu meinem ersten Seminar an der Universität. Mit mir waren 32 Personen (die maximale Teilnehmeranzahl) dafür angemeldet. In meiner Abiturklasse waren wir 26 Lernende und damit eine der größten Klassen an der Schule.

Fünf Minuten vor Seminarbeginn betrat ich den Raum, in dem jetzt schon fast alle Stühle belegt waren. Die Tische waren in einem $U$ angeordnet. Ich erinnerte mich an meinen ehemaligen Klassenraum, der fast genauso aussah, nur etwas größer. Um auf einen der zwei freien Stühle im hinteren Bereich des Raumes zu gelangen, musste ich entweder rechts oder links an den Tischreihen vorbeigehen. Der Durchgang war so eng, dass alle bereits Sitzenden ihren Stuhl vorrücken, ihre Taschen wegräumen und ihre Köpfe einziehen mussten, damit ich niemanden beim Vorbeigehen anstieß. Genauso ging es einem Kommilitonen, der zur gleichen Zeit wie ich eintraf und auf der anderen Seite versuchte zu dem letzten freien Stuhl zu gelangen. Alle, die den Raum danach betraten (ich zählte bereits fünf Personen), mussten sich Stühle aus dem Nebenraum holen und eine zweite Reihe hinter der letzten Reihe bilden oder sich in die Mitte des Raumes setzen. Beim Durchzählen kam ich jetzt auf 30 Personen, das heißt, es mussten noch zwei weitere Personen einen Platz finden. Mit der Seminarleitung kamen noch drei weitere Studierende in den Raum, die erst im Nachrückverfahren eine Studienzusage erhalten hatten und auch gerne am Seminar teilnehmen wollten.

So begann meine erste Uni-Veranstaltung mit 34 Mitstudierenden in einem Raum, der für höchstens 25 Lernende ausgelegt sein konnte. Beim Herausholen meines Notizblocks stieß ich zuerst meine rechte, dann meine linke Sitznachbarin an und versuchte anschließend, mich so wenig wie möglich zu bewegen. Die Studierenden in der „zweiten Reihe“ hinter mir, hatten kaum Sicht nach vorn und beschäftigten sich bereits mit ihren Smartphones als ich mich das zweite Mal umsah. Die vier Studierenden in der Mitte versuchten sich so klein wie möglich zu machen, um die Studierenden darum herum nicht zu stören oder 
vielleicht um die Aufmerksamkeit von 32 Augenpaaren im Rücken nicht zusätzlich auf sich zu lenken. Die Beteiligung im Seminar war sehr gering.

Ich hatte das Gefühl, dass eine angespannte Atmosphäre im Raum lag, die von Unsicherheit der Studierenden, geringem Handlungsspielraum der Dozentin und schlechter Luft geprägt war und sich durch die gesamte Veranstaltung zog.

\section{Flexible Nutzung und Nutzendenzentrierung}

Die Lernräume sollen den unterschiedlichen studentischen Bedürfnissen entsprechen und Einzel- sowie Gruppenlernplätze und Raum für Austausch bereitstellen. Räume sollen variabel genutzt werden können. Ein kreatives und produktives Arbeiten der vielen studentischen Gruppen und Initiativen soll ermöglicht werden. Der Fokus muss voll und ganz auf der variablen Nutzung liegen, sodass auch mit begrenzter Fläche sowohl Plätze zum Studieren und Austauschen vorhanden sind, als auch studentische Gruppen in den Räumen kreativ und produktiv werden können.

Die Raumgestaltung außerhalb von Veranstaltungsräumen ist ebenfalls ein zentrales Thema, da laut der Studie „Gesundheit Studierender in Deutschland 2017“ des Deutschen Zentrums für Hochschul- und Wissenschaftsforschung, der Freien Universität Berlin und der Techniker Krankenkasse ein Viertel der Studierenden ein erhöhtes Stresserleben angeben (Grützmacher et al. 2018, 48). Da Studierende häufig viele Freistunden zwischen ihren Veranstaltungen haben, verbringen sie viel Zeit an den Hochschulen, die unter Umständen nicht sinnvoll genutzt werden kann. Die Raumstruktur an Hochschulen sollte so angepasst sein, dass diese Zeitfenster effektiv für das Selbststudium genutzt werden können, aber auch Erholung möglich ist. Dafür sind Räume nötig, in denen eine geringe Lautstärke und wenig Ablenkung, zum Beispiel durch vorbeilaufende Personen, vorherrschen.

\section{Ausstattung}

Für die Zukunft einer partizipativen Hochschule müssen Lehr- und Lernräume vorhanden sein, die in erster Linie eine Ausstattung besitzen, die nicht neu erscheint und trotzdem teilweise nicht vorhanden ist. Dazu zählen Fenster, die die Möglichkeit bieten Tageslicht herein zu lassen, aber ebenfalls durch Rollos oder Vorhänge Schutz vor störender Sonneneinstrahlung bieten. Das Klima soll- 
te der Jahreszeit entsprechend reguliert werden können. Die Räume sollten Internetzugang haben, sowohl über WLAN für mobile Endgeräte, als auch über den gegebenenfalls im Raum fest installierten Computer. Dies sind wichtige Voraussetzungen dafür, dass die oben bereits genannte Digitalisierung von Lehr-Lernprozessen aktiv umgesetzt werden kann und nicht durch technische Schwierigkeiten behindert wird. Es sollten ebenfalls ausreichend Steckdosen an den Tischen vorhanden sein.

\section{Ausblick}

Die Hochschulen sind in der Umsetzung der in diesem Text beschriebenen Anforderungen an Lehr- und Lernräume gefragt. Dies ist eine Voraussetzung dafür, dass Studierende erfolgreich an Hochschulen lernen und sich bilden können. Damit die Lernenden aktiv ihre eigenen Lern- und Bildungsprozesse gestalten können, sind selbstverständlich nicht nur die Räume ausschlaggebend, denn dazu gehören viele weitere Aspekte, die beachtet werden müssen. Nichtsdestotrotz können Lehr- und Lernräume einen Beitrag dazu leisten, dass studentische Partizipation in der Lehre und somit auch die Qualität von Lehr-Lernprozessen gesteigert werden.

Ein Online-Self-Assessment (OSA), in dem getestet werden kann, inwiefern Lehr- und Lernräume partizipativ gestaltet sind und Sensibilisierung für eine partizipative Gestaltung entwickelt werden kann, ist zum Beispiel an der Universität Hamburg ${ }^{1}$ zu finden.

\section{Literatur}

Bergmann, J.; Sams, A. (2012): Flip your Classroom: Reach every student in every class every day. London: International Society for Technology in Education.

Günther, C.; Rauber, C. (2019): Ausbildung von Querdenkern. Bauliche Infrastruktur. Ein Weg zur Inklusion. HIS-HE: Medium 3. https://his-he.de/publikationen/detail/publikation/ ausbildung-von-querdenkern/.

Grützmacher, J.; Gusy, B.; Lesener, T.; Sudheimer, S.; Willige, J. (2018): Gesundheit Studierender in Deutschland 2017. Ein Kooperationsprojekt zwischen dem Deutschen Zentrum für Hochschul-und Wissenschaftsforschung, der Freien Universität Berlin und der Techniker Krankenkasse. https://www.ewi-psy.fu-berlin.de/einrichtungen/arbeitsbereiche/ppg/

$\overline{1 \mathrm{https}: / / \text { studpart.check.uni-hamburg.de/partizipative-lehr-lernraeume/. }}$ 
bwb-2017/_inhaltselemente/faktenblaetter/Gesamtbericht-Gesundheit-Studierender-inDeutschland-2017.pdf.

Leuphana (2019): Leitbild der Universität Leuphana. https://www.leuphana.de/universitaet/ leitbild.html.

Mayrberger, K: (2017): Partizipatives Lernen in der Online-Lehre. Anspruch, Konzept und Ausblick. In: H. R. Griesehop; E. Bauer (Hrsg.): Lehren und Lernen online. Lehr- und Lernerfahrungen im Kontext akademischer Online-Lehre. Wiesbaden: Springer Fachmedien, 109-129.

Siebert, H. (2006): Stichwort „Lernorte“. DIE Zeitschrift für Erwachsenenbildung 4, 20-21. https://www.die-bonn.de/zeitschrift/42006/siebert06_01.htm.

Stifterverband für die Deutsche Wissenschaft (2019): Hochschul-Bildungs-Report 2020. Essen: Stifterverband. http://www.hochschulbildungsreport2020.de/.

Universität Hamburg (2018): Leitbild der Universität Hamburg. https://www.uni-hamburg.de/ uhh/profil/leitbild.html.

Ziegele, F.; Neubert, P.; Mordhorst, L. (2019): Die Hochschule der Zukunft. Fels in der Brandung? Hochschulsport 2, 20-22. 
